hep-th/0610161

\title{
Wrapped M5-branes leading to five dimensional 2-branes
}

\author{
Moataz H. Emam1 \\ Department of Physics \\ Clark University \\ Worcester, MA 01610
}

\begin{abstract}
We study the dimensional reduction of M5-branes wrapping special Lagrangian 3-cycles of a Calabi-Yau manifold and show explicitly that they result in 2-branes coupled to the hypermultiplets of ungauged $\mathcal{N}=2 D=5$ supergravity theory. In addition to confirming previously known results, the calculation proves the relationship between them and provides further insight on how the topological properties of the compact space affect the lower dimensional fields.
\end{abstract}

\footnotetext{
${ }^{1}$ Electronic address: memam@clarku.edu
} 


\section{Introduction}

Black branes satisfying the Bogomol'nyi-Prasad-Sommerfield (BPS) condition have been studied from a variety of perspectives many times over the years, ever since it was discovered that such branes preserve some degree of supersymmetry [1]. Of particular interest are brane configurations wrapping manifolds with special or restricted holonomy. Such manifolds have been catalogued by Berger [2] and shown to admit calibrated forms (an excellent review is [3]). This allows for the construction of wrapped configurations simply by taking into account calibrated forms on the compact space (see [4] or [5] for detailed reviews and reference lists). Such a program was started by the realization [6] that certain constructions describing localized intersecting M5 branes [7] admit generalized Kähler calibrations, which take into account the flux of the eleven dimensional 4-form gauge field. This was further extended in the same reference [6] and used to find more wrapped brane configurations. Inevitably, branes wrapping other types of calibrated cycles were sought after using a variety of techniques. Of interest to us are branes wrapped over special Lagrangiancalibrated (SLAG) submanifolds. The first such solution was found in [4], and later published in [8]. A more general SLAG calibrated construction was announced in [9]. In the same reference, branes wrapping submanifolds with $G_{2}$ holonomy were also studied. Later, Fayyazuddin and Husain [10] took a second look at SLAG wrapped branes and proceeded to explore other instances of SLAG wrappings [11, 12].

On the other hand, it is also possible to argue that M-branes wrapping calibrated cycles of a Calabi-Yau manifold dimensionally reduce to interesting BPS configurations in lower dimensions. This has always been an assumption based on geometric and physical arguments. For example, the Kähler-calibrated branes of [6] dimensionally reduce to black holes and strings coupled to the vector multiplets of $\mathcal{N}=2 D=5$ supergravity as demonstrated in [13]. In addition to being formal proofs of hypotheses that have always been simply assumed, such calculations provide deeper insights into how lower dimensional fields arise as consequences of the topology of the compact subspace.

The other fields sector of ungauged $D=5$ supergravity theory is the hypermultiplets sector. A particular special case of that is the so-called universal hypermultiplet. This is better understood from a higher dimensional viewpoint as the dimensional reduction over SLAG cycles of a CalabiYau submanifold with constant complex structure moduli (a lightning review is presented in the next section). In [8], we found explicit SLAG-wrapped solutions in $D=11$ and showed how such wrappings dimensionally reduce to 2 -branes in $D=5$ coupled only to the universal hypermultiplet 
fields. In the same paper we analyzed the conditions on a more general $D=52$-brane that couples to the full set of hypermultiplets. From the higher dimensional perspective, this is assumed to be a M5-brane wrapping SLAG cycles of a Calabi-Yau 3-fold with non-trivial complex structure moduli; the same calibrated brane configuration studied by [9] and [10]. This correspondence has been assumed without proof in most of these references. In this paper, we provide that proof and show that at a scale much larger than the size of the compact space, one does indeed retrieve the results of [8].

The paper is structured as follows: Section 2 reviews how $D=11$ supergravity reduces to five dimensions, producing the ungauged $\mathcal{N}=2$ theory, and sets the conventions and notation. We are only interested in the hypermultiplets, so the vector multiplets sector will be ignored. Section 3 presents the eleven dimensional SLAG-wrapped M5-brane in the form of [10] as well as the 2brane of [8]. At the risk of confusing the reader, we had to change the notations used in the two references slightly in order to avoid using the same symbols to describe different things. Section 4 details the dimensional reduction of the eleven dimensional brane to produce the five dimensional result exactly and analyzes the geometric meaning of the $D=11$ SUSY conditions in the far field limit. Finally we conclude and propose further study.

\section{Dimensional reduction of $D=11$ supergravity}

Dimensionally reducing $D=11$ supergravity on a Calabi-Yau 3 -fold $\mathcal{M}$ yields ungauged $D=5$ $\mathcal{N}=2$ supergravity coupled to $\left(h_{1,1}-1\right)$ vector multiplets and $\left(h_{2,1}+1\right)$ hypermultiplets [14]; the $h$ 's being the Hodge numbers of $\mathcal{M}$. M-branes wrapping Kähler calibrated cycles of $\mathcal{M}$ deform the Kähler structure of $\mathcal{M}$ and reduce to configurations in which the vector multiplets are excited [13]. SLAG wrapped M-branes, our focus in this paper, deform the complex structure of $\mathcal{M}$ and reduce to configurations carrying charge under the hypermultiplet scalars. The two sectors of the theory decouple and we only keep the hypermultiplets in our presentation.

The $D=5 \mathcal{N}=2$ supergravity Langrangian including the full set of $\left(h_{2,1}+1\right)$ hypermultiplets can be written in terms of geometric quantities on the moduli space of the complex structures of the Calabi-Yau manifold $\mathcal{M}$. These structures are discussed in detail in [15] and we will give a brief review here. Start by taking a basis of the homology 3 -cycles $\left(A^{I}, B_{J}\right)$ with $I, J=0,1, \ldots, h_{2,1}$ 
and a dual cohomology basis of 3 -forms $\left(\alpha_{I}, \beta^{J}\right)$ such that

$$
\int_{A^{J}} \alpha_{I}=\int_{\mathcal{M}} \alpha_{I} \wedge \beta^{J}=\delta_{I}^{J}, \quad \int_{B_{I}} \beta^{J}=\int_{\mathcal{M}} \beta^{J} \wedge \alpha_{I}=-\delta_{I}^{J} .
$$

Define the periods of the holomorphic 3 -form $\Omega$ on $\mathcal{M}$ by

$$
Z^{I}=\int_{A^{I}} \Omega, \quad F_{I}=\int_{B_{I}} \Omega
$$

The periods $Z^{I}$ can be regarded as coordinates on the complex structure moduli space. Since $\Omega$ can be multiplied by an arbitrary complex number without changing the complex structure, the $Z^{I}$ are projective coordinates. The remaining periods $F_{I}$ can then be regarded as functions $F_{I}(Z)$. One can further show that $F_{I}$ is the gradient of a function $F(Z)$, known as the prepotential, that is homogeneous of degree two in the coordinates, i.e. $F_{I}=\partial_{I} F(Z)$ with $F(\lambda Z)=\lambda^{2} F(Z)$. The quantity $F_{I J}(Z)=\partial_{I} \partial_{J} F(Z)$ will also play an important role. Non-projective coordinates can then be given by taking e.g. $z^{i}=Z^{i} / Z^{0}$ with $i=1, \ldots, h_{2,1}$. The Kähler potential of the complex structure moduli space is $\mathcal{K}=-\ln \left(i \int_{\mathcal{M}} \Omega \wedge \bar{\Omega}\right)$. Given the expansion of $\Omega$ in terms of the periods

$$
\Omega=Z^{I} \alpha_{I}-F_{I} \beta^{I}
$$

the Kähler potential is determined in terms of the prepotential $F(Z)$ according to

$$
\mathcal{K}=-\ln \left[i\left(Z^{I} \bar{F}_{I}-\bar{Z}^{I} F_{I}\right)\right]
$$

The so-called period matrix $\mathcal{N}_{I J}$ is defined by

$$
\mathcal{N}_{I J}=\bar{F}_{I J}-2 i \frac{N_{I K} Z^{K} N_{J L} Z^{L}}{Z^{P} N_{P Q} Z^{Q}}=\theta_{I J}-i \gamma_{I J}
$$

where $N_{I J}=\operatorname{Im}\left(F_{I J}\right), \gamma^{I J} \gamma_{J K}=\delta_{K}^{I}$, and $(\theta, \gamma)$ are real matrices.

The derivation of the Lagrangian for the bosonic fields of the $D=5$ theory is sketched in [16] and detailed in [4]. The bosonic part of the $D=11$ action is the familiar:

$$
S_{11}=\frac{1}{2 \kappa_{11}^{2}} \int d^{11} x \sqrt{-G}\left(R-\frac{1}{48} F^{2}\right)-\frac{1}{12 \kappa_{11}^{2}} \int A \wedge F \wedge F
$$

where $F$ is given by $F=d A ; A$ being the usual eleven dimensional 3 -form gauge field. The dimensional reduction of (6) is done over the metric:

$$
\begin{aligned}
& d s^{2}=e^{2 \sigma / 3} g_{\mu \nu} d x^{\mu} d x^{\nu}+e^{-\sigma / 3} k_{\tilde{I} \tilde{J}} d x^{\tilde{I}} d x^{\tilde{J}} \\
& \mu, \nu=0, \ldots, 4 \quad \tilde{I}, \tilde{J}=1, \ldots, 6
\end{aligned}
$$


where $k_{\tilde{I} \tilde{J}}$ is a fixed Ricci flat metric on the Calabi-Yau space $\mathcal{M}$. The $D=113$-form is expanded in terms of the cohomology basis as follows

$$
A=\frac{1}{3 !} A_{\mu \nu \rho} d x^{\mu} \wedge d x^{\nu} \wedge d x^{\rho}+\sqrt{2}\left(\zeta^{I} \alpha_{I}+\tilde{\zeta}_{I} \beta^{I}\right)
$$

and

$$
\begin{aligned}
F=d A & =\frac{1}{4 !} F_{\mu \nu \rho \sigma} d x^{\mu} \wedge d x^{\nu} \wedge d x^{\rho} \wedge d x^{\sigma} \\
& +\sqrt{2}\left[\left(\partial_{\mu} \zeta^{I}\right) \alpha_{I}+\left(\partial_{\mu} \tilde{\zeta}_{I}\right) \beta^{I}\right] \wedge d x^{\mu}
\end{aligned}
$$

The resulting $D=5$ bosonic action is

$$
\begin{aligned}
S_{5} & =\frac{1}{2 \kappa_{5}^{2}} \int d^{5} x \sqrt{-g}\left[R-\frac{1}{2}\left(\partial_{\mu} \sigma\right)\left(\partial^{\mu} \sigma\right)-G_{i \bar{j}}\left(\partial_{\mu} z^{i}\right)\left(\partial^{\mu} z^{\bar{j}}\right)\right. \\
& \left.-\frac{1}{48} e^{-2 \sigma} F_{\mu \nu \rho \sigma} F^{\mu \nu \rho \sigma}-\frac{1}{24} \varepsilon_{\mu \nu \rho \sigma \alpha} F^{\mu \nu \rho \sigma} K^{\alpha}(\zeta, \tilde{\zeta})+e^{\sigma} L_{\mu}^{\mu}(\zeta, \tilde{\zeta})\right],
\end{aligned}
$$

where we have defined:

$$
\begin{aligned}
K_{\alpha}(\zeta, \tilde{\zeta})= & {\left[\zeta^{I}\left(\partial_{\alpha} \tilde{\zeta}_{I}\right)-\tilde{\zeta}_{I}\left(\partial_{\alpha} \zeta^{I}\right)\right] } \\
L_{\mu \nu}(\zeta, \tilde{\zeta})= & -\left(\gamma_{I J}+\gamma^{K L} \theta_{I K} \theta_{J L}\right)\left(\partial_{\mu} \zeta^{I}\right)\left(\partial_{\nu} \zeta^{J}\right)-\gamma^{I J}\left(\partial_{\mu} \tilde{\zeta}_{I}\right)\left(\partial_{\nu} \tilde{\zeta}_{J}\right) \\
& -2 \gamma^{I K} \theta_{K J}\left(\partial_{\mu} \zeta^{J}\right)\left(\partial_{\nu} \tilde{\zeta}_{I}\right)
\end{aligned}
$$

The scalar fields $z^{i}, z^{\bar{i}}$ with $i=1, \ldots, h_{2,1}$ are complex coordinates on the complex structure moduli space with metric $G_{i \bar{j}}=\partial_{i} \partial_{\bar{j}} \mathcal{K}$. The pseudo-scalar axions $\left(\zeta^{I}, \tilde{\zeta}_{I}\right)$ arise from the dimensional reduction of the $D=113$-form gauge potential. The scalar field $\sigma$ is the overall volume scalar of $\mathcal{M}$ and $F_{\mu \nu \rho \sigma}$ is the $D=54$-form field strength. Each hypermultiplet has 4 scalar fields. The scalars $\left(z^{i}, z^{\bar{i}}, \zeta^{i}, \tilde{\zeta}_{i}\right)$ make up $h_{2,1}$ of the hypermultiplets, while the additional universal hypermultiplet is comprised of the fields $\left(a, \sigma, \zeta^{0}, \tilde{\zeta}_{0}\right)$, where the so-called universal axion $a$ is the scalar dual to the 3-form gauge potential $A_{\mu \nu \rho}$.

Further study of the structure of the theory (see [16] and the references within) reveals that the hypermultiplets define a $\left(h_{2,1}+1\right)$-dimensional quaternionic space. This structure, in five dimensions, is dual to the special Kähler geometry of the $D=4$ vector multiplets sector via the so called c-map (e.g. [17]). This duality justifies the use of special Kähler geometry techniques as opposed to the explicit quaternionic form.

Furthermore, one finds that the theory is invariant under the symplectic group $\operatorname{Sp}\left(2 h_{2,1}, \mathbb{R}\right)$, i.e. (10) actually defines a family of Lagrangians that differ from each other only by a rotation in 
symplectic space that has no effect on the physics. In fact, if we define

$$
V=\left(\begin{array}{c}
L^{I} \\
M_{J}
\end{array}\right) \equiv e^{\mathcal{K} / 2}\left(\begin{array}{c}
Z^{I} \\
F_{J}
\end{array}\right)
$$

satisfying

$$
\nabla_{\bar{i}} V=\left[\partial_{\bar{i}}-\frac{1}{2}\left(\partial_{\bar{i}} \mathcal{K}\right)\right] V=0
$$

then $V$ is a basis vector in symplectic space that satisfies the inner product

$$
i\langle V \mid \bar{V}\rangle=i\left(\bar{L}^{I} M_{I}-L^{I} \bar{M}_{I}\right)=1
$$

An orthogonal vector may be defined by

$$
U_{i} \equiv\left(\begin{array}{c}
f_{i}^{I} \\
h_{J \mid i}
\end{array}\right)=\nabla_{i} V
$$

such that

$$
\left\langle V \mid U_{i}\right\rangle=\left\langle V \mid U_{\bar{i}}\right\rangle=0
$$

Based on this, the following identities may be derived:

$$
\begin{aligned}
\mathcal{N}_{I J} L^{J} & =M_{I}, \quad \mathcal{N}_{I J} f_{i}^{J}=h_{I \mid i} \\
\left(\nabla_{\bar{j}} f_{i}^{I}\right) & =G_{i \bar{j}} L^{I}, \quad\left(\nabla_{\bar{j}} h_{i I}\right)=G_{i \bar{j}} M_{I} \\
\gamma_{I J} L^{I} \bar{L}^{J} & =\frac{1}{2}, \quad G_{i \bar{j}}=2 f_{i}^{I} \gamma_{I J} f_{\bar{j}}^{J},
\end{aligned}
$$

as well as the very useful:

$$
\begin{aligned}
\gamma^{I J} & =2\left(G^{i \bar{j}} f_{i}^{I} f_{\bar{j}}^{J}+L^{I} \bar{L}^{J}\right) \\
\left(\gamma_{I J}+\gamma^{K L} \theta_{I K} \theta_{J L}\right) & =2\left(G^{i \bar{j}} h_{i I} h_{\bar{j} J}+M_{I} \bar{M}_{J}\right) \\
\gamma^{I K} \theta_{K J} & =2\left(L^{I} \bar{M}_{J}+\bar{L}^{I} M_{J}\right) .
\end{aligned}
$$

Such detail follows directly from the topology of the underlying compact manifold, and it is indeed a wonder that we can understand so much about it with little need for the explicit form of a metric on $\mathcal{M}$. 


\section{$3 \quad$ Wrapped M5-branes and $D=5$ 2-branes with hypermultiplets}

The proposition we are attempting to analyze in this paper is that M5-branes wrapping SLAG cycles of a CY 3-fold dimensionally reduce to 2-branes coupled to the hypermultiplets of $\mathcal{N}=2$ $D=5$ ungauged SUGRA. In this section we summarize both constructions as they were presented in references [10] and [8].

\subsection{The $D=5$ 2-brane}

Based on the notation established in 92 the $D=5$ 2-brane spacetime metric coupled to the hypermultiplets may be written as follows [8]:

$$
d s^{2}=\left(-d t^{2}+d x_{1}^{2}+d x_{2}^{2}\right)+e^{-2 \sigma} \delta_{a b} d x^{a} d x^{b}, \quad a, b=3,4
$$

We define a number $\left(h_{2,1}+1\right)$ of harmonic functions

$$
H_{I}=h_{I}+q_{I} \ln r \quad, \quad \tilde{H}^{I}=\tilde{h}^{I}+\tilde{q}^{I} \ln r \quad, \quad I=0, \ldots, h_{2,1},
$$

where $h$ and $\tilde{h}$ are constants, $r$ is the radial coordinate in the two dimensional space transverse to the brane and $(q, \tilde{q})$ are electric and magnetic charges. The SUSY equations yield the following constraints on the scalar fields:

$$
\begin{aligned}
\left(\partial_{a} \sigma\right) & =-2 e^{\frac{\sigma}{2}}\left[L^{I}\left(\partial_{a} H_{I}\right)-M_{I}\left(\partial_{a} \tilde{H}^{I}\right)\right] \\
& =-2 e^{\frac{\sigma}{2}}\left[\bar{L}^{I}\left(\partial_{a} H_{I}\right)-\bar{M}_{I}\left(\partial_{a} \tilde{H}^{I}\right)\right] \\
\left(\partial_{a} z^{i}\right) & =-e^{\frac{\sigma}{2}} G^{i \bar{j}}\left[f_{\bar{j}}^{I}\left(\partial_{a} H_{I}\right)-h_{\bar{j} I}\left(\partial_{a} \tilde{H}^{I}\right)\right] \\
\left(\partial_{a} z^{\bar{i}}\right) & =-e^{\frac{\sigma}{2}} G^{\bar{i} j}\left[f_{j}^{I}\left(\partial_{a} H_{I}\right)-h_{j I}\left(\partial_{a} \tilde{H}^{I}\right)\right] \\
\left(\partial_{a} \zeta^{I}\right) & = \pm \varepsilon_{a}^{c}\left(\partial_{c} \tilde{H}^{I}\right) \\
\left(\partial_{a} \tilde{\zeta}_{I}\right) & = \pm \varepsilon_{a}{ }^{c}\left(\partial_{c} H_{I}\right),
\end{aligned}
$$

and $F_{\mu \nu \rho \sigma}=0$. Using a well-known relationship between the charges $q$ and $\tilde{q}$ and the central charge $Z$ of the theory as follows [18]:

$$
\begin{aligned}
& Z=\left(L^{I} q_{I}-M_{I} \tilde{q}^{I}\right) \\
& \bar{Z}=\left(\bar{L}^{I} q_{I}-\bar{M}_{I} \tilde{q}^{I}\right)
\end{aligned}
$$


equations (25126) may be simplified to:

$$
\begin{aligned}
\frac{d \sigma}{d r} & =-2 e^{\frac{\sigma}{2}} \frac{Z}{r} \\
\frac{d z^{i}}{d r} & =-e^{\frac{\sigma}{2}} \frac{\nabla^{i} \bar{Z}}{r} \\
\frac{d z^{\bar{i}}}{d r} & =-e^{\frac{\sigma}{2}} \frac{\nabla^{\bar{i}} Z}{r},
\end{aligned}
$$

which may further be shown to satisfy [19]:

$$
H_{I}=i\left(F_{I}-\bar{F}_{I}\right) \quad \tilde{H}^{I}=i\left(Z^{I}-\bar{Z}^{I}\right) .
$$

\subsection{The wrapped M5-brane}

The spacetime metric derived by Fayyazuddin and Husain in reference [10] may be written as follows:

$$
\begin{aligned}
& d s^{2}=H^{-1 / 3}\left(-d t^{2}+d x_{1}^{2}+d x_{2}^{2}\right)+g_{\tilde{I} \tilde{J}} d x^{\tilde{I}} d x^{\tilde{J}}+H^{2 / 3} \delta_{a b} d x^{a} d x^{b} \\
& \tilde{I}, \tilde{J}=1, \ldots, 6 \quad a, b=3,4
\end{aligned}
$$

representing a M5-brane wrapping a Calabi-Yau 3-fold with metric $g_{\tilde{I} \tilde{J}}$. The brane's tension distorts the compact space such that it is no longer strictly Calabi-Yau. The scale factor $H$ is a function in the two dimensional transverse space.

The brane is naturally coupled to the eleven dimensional 7-form field strength which was constructed in the same reference. For our purposes, its dual 4-form field strength (which they also gave) may be more useful. It is:

$$
\begin{aligned}
F_{(4)} & =\frac{1}{4} H^{1 / 6} \varepsilon_{a b} \star_{6} d_{6}\left[H^{1 / 2}(\operatorname{Re} \Omega)\right] \wedge d x^{a} \wedge d x^{b} \\
& -\frac{i}{2} H^{-1 / 2} \varepsilon_{a}{ }^{b} \partial_{b}\left[H^{1 / 2}(\operatorname{Im} \Omega)\right] \wedge d x^{a}
\end{aligned}
$$

where $\Omega$ is a globally defined holomorphic form which turns out to be the usual Calabi-Yau 3-form and $\star_{6}$ is the Hodge dual operator on $\mathcal{M}$.

Dictated by SUSY preservation, certain constraints on the compact manifold were also found:

$$
\begin{aligned}
\bar{\Omega} \wedge{ }_{\star_{6}} d_{6} \Omega & =0 \\
d_{6}(\Omega-\bar{\Omega}) & =0 \\
\operatorname{det}\left(g_{\tilde{I} \tilde{J}}\right) \equiv g & =H \\
\Omega^{\tilde{I} \tilde{J} \tilde{K}}\left(\partial_{a} \bar{\Omega}_{\tilde{I} \tilde{J} \tilde{K}}\right) & =12 \partial_{a} \ln g .
\end{aligned}
$$




\section{Dimensional reduction and analysis}

We now show that the $D=52$-brane of $\$ 3.1$ is the dimensional reduction of the SLAG-wrapped M5-brane of $₫ 3.2$. Since we already have a dimensional reduction scheme, as given in $₫ 2$, one can simply merge the $D=11$ equations into that scheme and see if what we retrieve is consistent with the $D=5$ results.

We begin by considering the metric (31). Rearranging:

$$
d s^{2}=H^{-1 / 3}\left(-d t^{2}+d x_{1}^{2}+d x_{2}^{2}+H \delta_{a b} d x^{a} d x^{b}\right)+g_{\tilde{I} \tilde{J}} d x^{\tilde{I}} d x^{\tilde{J}},
$$

and comparing with the form of the metric (17) used for the dimensional reduction of the theory, one is forced to conclude that:

$$
H=e^{-2 \sigma}, \quad g_{\tilde{I} \tilde{J}}=H^{1 / 6} k_{\tilde{I} \tilde{J}}=e^{-\sigma / 3} k_{\tilde{I} \tilde{J}}
$$

Based on this, one immediately sees that the five dimensional metric is:

$$
\begin{aligned}
g_{\mu \nu} d x^{\mu} d x^{\nu} & =\left(-d t^{2}+d x_{1}^{2}+d x_{2}^{2}\right)+H \delta_{a b} d x^{a} d x^{b} \\
& =\left(-d t^{2}+d x_{1}^{2}+d x_{2}^{2}\right)+e^{-2 \sigma} \delta_{a b} d x^{a} d x^{b}
\end{aligned}
$$

which is exactly the $D=5$ result (23).

Next, we turn to the field strength. To begin with, we argue that as the compact subspace is shrunk to a point, variations on $\mathcal{M}$ vanish and expressions such as $d_{6}\left[H^{1 / 2}(\operatorname{Re} \Omega)\right]$ can be neglected, so we set the first term of (32) to zero. We also identify $\Omega$ as the holomorphic Calabi-Yau 3-form. To facilitate the calculation, we make the assumption that $\sigma=\mathcal{K}$, where the Kähler potential $\mathcal{K}$ is defined by (4) 2 , and make use of (3) as follows:

$$
\begin{aligned}
\Omega & =\left(Z^{I} \alpha_{I}-F_{I} \beta^{I}\right)=e^{-\sigma / 2}\left(L^{I} \alpha_{I}-M_{I} \beta^{I}\right) \text { and } c . c . \\
\left(\partial_{a} \Omega\right) & =e^{-\sigma / 2}\left[\left(\partial_{a} L^{I}\right) \alpha_{I}-\left(\partial_{a} M_{I}\right) \beta^{I}\right]-\frac{1}{2} e^{-\sigma / 2}\left(\partial_{a} \sigma\right)\left(L^{I} \alpha_{I}-M_{I} \beta^{I}\right) \text { and c.c. }
\end{aligned}
$$

where c.c. means the complex conjugate of each of these equations. We find:

$$
\begin{aligned}
F & =-\frac{i}{4} \varepsilon_{a}{ }^{b}\left[\left(\partial_{b} \Omega\right)-\left(\partial_{b} \bar{\Omega}\right)\right] \wedge d x^{a}+\frac{i}{4} \varepsilon_{a}{ }^{b}\left(\partial_{b} \sigma\right)[\Omega-\bar{\Omega}] \wedge d x^{a} \\
& =\frac{i}{4} \varepsilon_{a}{ }^{b} e^{-\sigma / 2}\left[-\left(\partial_{b} L^{I}\right)+\left(\partial_{b} \bar{L}^{I}\right)-\frac{1}{2}\left(\partial_{b} \sigma\right) L^{I}+\frac{1}{2}\left(\partial_{b} \sigma\right) \bar{L}^{I}\right] \alpha_{I} \wedge d x^{a} \\
& +\frac{i}{4} \varepsilon_{a}{ }^{b} e^{-\sigma / 2}\left[\left(\partial_{b} M_{I}\right)-\left(\partial_{b} \bar{M}_{I}\right)+\frac{1}{2}\left(\partial_{b} \sigma\right) M_{I}-\frac{1}{2}\left(\partial_{b} \sigma\right) \bar{M}_{I}\right] \beta^{I} \wedge d x^{a} .
\end{aligned}
$$

\footnotetext{
${ }^{2}$ This is essentially the assumption made by [19] in the context of solving the attractor equations of four dimensional black holes coupled to the vector multiplets.
} 
It is straightforward to show that [4]:

$$
\left(\partial_{b} L^{I}\right)=f_{i}^{I}\left(\partial_{b} z^{i}\right), \quad\left(\partial_{b} M_{I}\right)=h_{i I}\left(\partial_{b} z^{i}\right) \text { and c.c. }
$$

For vanishing universal axion, equation (9) becomes

$$
F=\sqrt{2}\left[\left(\partial_{a} \zeta^{I}\right) \alpha_{I}+\left(\partial_{a} \tilde{\zeta}_{I}\right) \beta^{I}\right] \wedge d x^{a}
$$

which we compare with (42) to conclude:

$$
\begin{aligned}
\left(\partial_{a} \zeta^{I}\right) & =\frac{i}{4 \sqrt{2}} \varepsilon_{a}{ }^{b} e^{-\sigma / 2}\left[-f_{i}^{I}\left(\partial_{b} z^{i}\right)+f_{\bar{i}}^{I}\left(\partial_{b} z^{\bar{i}}\right)-\frac{1}{2}\left(\partial_{b} \sigma\right) L^{I}+\frac{1}{2}\left(\partial_{b} \sigma\right) \bar{L}^{I}\right] \\
\left(\partial_{a} \tilde{\zeta}_{I}\right) & =\frac{i}{4 \sqrt{2}} \varepsilon_{a}{ }^{b} e^{-\sigma / 2}\left[h_{i I}\left(\partial_{b} z^{i}\right)-h_{\bar{i} I}\left(\partial_{b} z^{\bar{i}}\right)+\frac{1}{2}\left(\partial_{b} \sigma\right) M_{I}-\frac{1}{2}\left(\partial_{b} \sigma\right) \bar{M}_{I}\right] .
\end{aligned}
$$

The crucial step is to insert the constraints (25) and (26) in their respective slots in (45/46) and see if we can retrieve (27). Doing so for (45) and rearranging:

$$
\begin{aligned}
\left(\partial_{a} \zeta^{I}\right) & =\frac{i}{4 \sqrt{2}} \varepsilon_{a}{ }^{b}\left[\left(f_{i}^{I} f_{\bar{j}}^{J} G^{i \bar{j}}+L^{I} \bar{L}^{J}\right)-\left(f_{j}^{J} f_{\bar{i}}^{I} G^{j \bar{i}}+L^{J} \bar{L}^{I}\right)\right]\left(\partial_{b} H_{J}\right) \\
& +\frac{i}{4 \sqrt{2}} \varepsilon_{a}{ }^{b}\left[-f_{i}^{I} h_{\bar{j} J} G^{i \bar{j}}-L^{I} \bar{M}_{J}+f_{\bar{i}}^{I} h_{j J} G^{\bar{i} j}+\bar{L}^{I} M_{J}\right]\left(\partial_{b} \tilde{H}^{J}\right) .
\end{aligned}
$$

The first term cancels out, while for the second we can use the identities (17) as well as the definition (5) to get:

$$
\begin{aligned}
\left(\partial_{a} \zeta^{I}\right)= & \frac{i}{4 \sqrt{2}} \varepsilon_{a}{ }^{b}\left[-\left(f_{i}^{I} f_{\bar{j}}^{K} G^{i \bar{j}}+L^{I} \bar{L}^{K}\right) \theta_{K J}+\left(f_{\bar{i}}^{I} f_{j}^{K} G^{\bar{i} j}+\bar{L}^{I} L^{K}\right) \theta_{K J}\right. \\
& \left.-i\left(f_{i}^{I} f_{\bar{j}}^{K} G^{i \bar{j}}+L^{I} \bar{L}^{K}\right) \gamma_{K J}-i\left(f_{\bar{i}}^{I} f_{j}^{K} G^{\bar{i} j}+\bar{L}^{I} L^{K}\right) \gamma_{K J}\right]\left(\partial_{b} \tilde{H}^{J}\right) .
\end{aligned}
$$

Once again, the terms in $\theta$ cancel out, while, using (20), the terms in $\gamma$ add up to:

$$
\left(\partial_{a} \zeta^{I}\right)=\frac{i}{4 \sqrt{2}} \varepsilon_{a}{ }^{b}\left(-i \gamma^{I K} \gamma_{K J}\right)\left(\partial_{b} \tilde{H}^{J}\right)=\frac{1}{4 \sqrt{2}} \varepsilon_{a}{ }^{b} \delta_{J}^{I}\left(\partial_{b} \tilde{H}^{J}\right)=\frac{1}{4 \sqrt{2}} \varepsilon_{a}{ }^{b}\left(\partial_{b} \tilde{H}^{I}\right),
$$

which, up to a difference in numerical constants due to different normalization conventions, is the expression (27) for $\left(\partial_{a} \zeta^{I}\right)$ found using the five dimensional equations! The calculation for $\left(\partial_{a} \tilde{\zeta}_{I}\right)$ is very similar, and is only different in the usage of (21) instead of (20) in the appropriate steps, giving:

$$
\left(\partial_{a} \tilde{\zeta}_{I}\right)=\frac{1}{4 \sqrt{2}} \varepsilon_{a}^{b}\left(\partial_{b} H_{I}\right)
$$

Finally, we look at the geometric significance of the constraints (33, 34, 35, 36). It is clear that in the far field limit, (33) and (34) vanish identically. Condition (35), on the other hand, may be 
understood as follows: From (38), one sees that $g=H k$, where $k \equiv \operatorname{det}\left(k_{\tilde{I} \tilde{J}}\right)$. From (35) one then concludes that $k=1$, i.e. constant, which is simply the statement that $k_{\tilde{I} \tilde{J}}$ is Ricci-flat. For the last condition (36), we proceed in the following way:

$$
\Omega^{\tilde{I} \tilde{J} \tilde{K}}\left(\partial_{a} \bar{\Omega}_{\tilde{I} \tilde{J} \tilde{K}}\right)=12\left(\partial_{a} \ln g\right)=12\left(\partial_{a} \ln H\right)=-24\left(\partial_{a} \sigma\right)
$$

Since the dilaton is a real field, then this is the statement that $\Omega^{\tilde{I} \tilde{J} \tilde{K}}\left(\partial_{a} \bar{\Omega}_{\tilde{I} \tilde{J} \tilde{K}}\right)$ is a real quantity. Then by setting its imaginary part equal to zero, we may proceed with a bit of algebra as follows:

$$
\begin{aligned}
\Omega^{\tilde{I} \tilde{J} \tilde{K}}\left(\partial_{a} \bar{\Omega}_{\tilde{I} \tilde{J} \tilde{K}}\right)=\operatorname{Re} \Omega^{\tilde{I} \tilde{J} \tilde{K}}\left(\partial_{a} \operatorname{Re} \bar{\Omega}_{\tilde{I} \tilde{J} \tilde{K}}\right)+\operatorname{Im} \Omega^{\tilde{I} \tilde{J} \tilde{K}}\left(\partial_{a} \operatorname{Im} \bar{\Omega}_{\tilde{I} \tilde{J} \tilde{K}}\right) & =-24\left(\partial_{a} \sigma\right) \\
& {\left[\bar{\Omega}^{\tilde{I} \tilde{J} \tilde{K}}\left(\partial_{a} \Omega_{\tilde{I} \tilde{J} \tilde{K}}\right)+\Omega^{\tilde{I} \tilde{J} \tilde{K}}\left(\partial_{a} \bar{\Omega}_{\tilde{I} \tilde{J} \tilde{K}}\right)\right]=-48\left(\partial_{a} \sigma\right) }
\end{aligned}
$$

Since

$$
\left[\bar{\Omega}^{\tilde{I} \tilde{J} \tilde{K}}\left(\partial_{a} \Omega_{\tilde{I} \tilde{J} \tilde{K}}\right)+\Omega^{\tilde{I} \tilde{J} \tilde{K}}\left(\partial_{a} \bar{\Omega}_{\tilde{I} \tilde{J} \tilde{K}}\right)\right]=\partial_{a}\left(\Omega^{\tilde{I} \tilde{J} \tilde{K}} \bar{\Omega}_{\tilde{I} \tilde{J} \tilde{K}}\right)=3 !\left(\partial_{a}|\Omega|^{2}\right)
$$

then

$$
\left(\partial_{a} \sigma\right)=-\frac{1}{8}\left(\partial_{a}|\Omega|^{2}\right) .
$$

In other words, (36) is simply the statement that the dilaton field is proportional to the norm of the Calabi-Yau 3-form, which is a constant on $\mathcal{M}$ but is not necessarily so at any point in the transverse space, due to the variation of the complex structure moduli of $\mathcal{M}$.

\section{Conclusion}

We have explicitly shown that the dimensional reduction of a M5-brane wrapping special Lagrangian 3-cycles of a Calabi-Yau manifold deforming the complex structure [10] excites only the hypermultiplets sector of ungauged five dimensional $\mathcal{N}=2$ supergravity. The $D=5$ universal axion $a$ (or its dual 3-form gauge field) vanishes and the result is a 2-brane coupled to the hypermultiplet fields [8]. This constitutes a proof of this relationship, often quoted in the literature, as well as further confirmation of both results. In addition, it provides further confirmation of the interpretation of [10] as a wrapped brane. This paper may be thought of as the sequel to an argument presented in [8] where a, much shorter, calculation has shown that a certain configuration of M-branes wrapping SLAG cycles of a Calabi-Yau with constant complex structure moduli excite only the universal hypermultiplet and result in a special case of the more general five dimensional 2-brane with full hypermultiplets discussed therein and here. 
Along with [13], our calculation provides more hints to open questions concerning compactification mechanisms, what classes of Calabi-Yau metrics are relevant and so on. Calculations such as these may also help analyze other brane configurations wrapping SLAG-calibrated cycles, as well as other supersymmetric cycles in spaces with restricted holonomy. For example, it would be interesting to see what lower dimensional results could arise from the dimensional reduction of M-branes wrapping manifolds with $G_{2}$ or $\operatorname{spin}(7)$ holonomy. More interesting than the result perhaps, as often happens, is the manner with which the result arises. We plan to explore this in future work.

\section{Acknowledgments}

We are grateful to Ansar Fayyazuddin and David Kastor for pointing out some subtleties in the calculation and for taking the time to read the manuscript and providing further advice.

\section{References}

[1] G. W. Gibbons and C. M. Hull, "A Bogomolny Bound For General Relativity And Solitons In N=2 Supergravity," Phys. Lett. B 109, 190 (1982).

[2] M. Berger, "Sur les groupes d'holonomie homogène des variétés à connexion affine et des variétés riemanniennes," Bull. Soc. Math. France 83, 225 (1955).

[3] D. Joyce, "Lectures on Calabi-Yau and special Lagrangian geometry," arXiv:math.dg/0108088.

[4] M. H. Emam, "Calibrated brane solutions of M-theory," arXiv:hep-th/0410100.

[5] T. Z. Husain, "If I only had a brane!," arXiv:hep-th/0304143].

[6] H. Cho, M. Emam, D. Kastor and J. H. Traschen, "Calibrations and Fayyazuddin-Smith spacetimes," Phys. Rev. D 63, 064003 (2001) arXiv:hep-th/0009062.

[7] A. Fayyazuddin and D. J. Smith, "Localized intersections of M5-branes and four-dimensional superconformal field theories," JHEP 9904, 030 (1999) arXiv:hep-th/9902210].

[8] M. H. Emam, "Five dimensional 2-branes from special Lagrangian wrapped M5-branes," Phys. Rev. D 71, 125020 (2005) arXiv:hep-th/0502112. 
[9] D. Martelli and J. Sparks, "G-structures, fluxes and calibrations in M-theory," Phys. Rev. D 68, 085014 (2003) arXiv:hep-th/0306225.

[10] A. Fayyazuddin and T. Z. Husain, "The geometry of M-branes wrapping special Lagrangian cycles," arXiv:hep-th/0505182.

[11] A. Fayyazuddin, T. Z. Husain and I. Pappa, "The geometry of wrapped M5-branes in CalabiYau 2-folds," Phys. Rev. D 73, 126004 (2006) arXiv:hep-th/0509018.

[12] A. Fayyazuddin and T. Z. Husain, "Calibrations, torsion classes and wrapped M-branes," Phys. Rev. D 73, 106007 (2006) arXiv:hep-th/0512030.

[13] D. Kastor, "From wrapped M-branes to Calabi-Yau black holes and strings," JHEP 0307, 040 (2003) arXiv:hep-th/0305261.

[14] A. C. Cadavid, A. Ceresole, R. D'Auria and S. Ferrara, "Eleven-dimensional supergravity compactified on Calabi-Yau threefolds," Phys. Lett. B 357, 76 (1995) arXiv:hep-th/9506144.

[15] P. Candelas and X. de la Ossa, "Moduli Space Of Calabi-Yau Manifolds," Nucl. Phys. B 355, 455 (1991).

[16] M. Gutperle and M. Spalinski, "Supergravity instantons for N = 2 hypermultiplets," Nucl. Phys. B 598, 509 (2001) arXiv:hep-th/0010192.

[17] J. De Jaegher, B. de Wit, B. Kleijn and S. Vandoren, "Special geometry in hypermultiplets," Nucl. Phys. B 514, 553 (1998) arXiv:hep-th/9707262.

[18] E. Witten and D. I. Olive, "Supersymmetry Algebras That Include Topological Charges," Phys. Lett. B 78, 97 (1978).

[19] W. A. Sabra, "Black holes in N = 2 supergravity theories and harmonic functions," Nucl. Phys. B 510, 247 (1998) arXiv:hep-th/9704147. 